\title{
A High-Speed Design Methodology for Inductive Coupling Links in 3D-ICs
}

\author{
Benjamin J. Fletcher*, Shidhartha Das ${ }^{\dagger}$, Terrence Mak* \\ *Department of Electronics and Computer Science University of Southampton, UK. ${ }^{\dagger}$ ARM Ltd, Cambridge, UK \\ *\{bjf1g13, tmak\}@ecs.soton.ac.uk, ${ }^{\dagger}$ Shidhartha.Das@arm.com
}

\begin{abstract}
Inductive coupling links (ICLs) are gaining traction as an alternative to through silicon vias (TSVs) for 3D integration, promising high-bandwidth connectivity without the inflated fabrication costs associated with TSV-enabled processes. For power-efficient ICL design, optimisation of the utilised physical inductor geometries is essential, however typically necessitates the use of finite element analysis (FEA) in addition to manual parameter fitting, a process that can take several hours even for a single geometry. As a result, the generation of optimised inductor designs poses a significant challenge. In this paper, we address this challenge, presenting a CAD-tool for Optimisation of Inductive coupling Links for 3D-ICs (COIL-3D ${ }^{1}$ ). COIL-3D uses a rapid solver based upon semi-empirical expressions to quickly and accurately characterise a given link, in conjunction with a high-speed refined optimisation flow to find optimal inductor geometries for use in ICL-based 3D-ICs. The proposed solver achieves an average accuracy within $9.1 \%$ of commercial FEA software tools, and the proposed optimisation flow reduces the search time by 26 orders of magnitude. This work unlocks new potential for power-efficient 3D integration using inductive coupling links.
\end{abstract}

\section{INTRODUCTION}

In recent years, 3D system integration has emerged as a promising 'more-than-Moore' technology whereby dies are stacked vertically; increasing device density, shortening interconnect lengths and hence enhancing the performance of ICs [1]. Typically, research surrounding 3D integration focuses on through silicon vias (TSVs) to provide electrical connections between dies, however, incorporating TSVs introduces many additional processing stages, resulting in inflated fabrication and testing costs, in addition to diminished yields [2].

An alternative solution, which overcomes these problems is the use of inductive coupling links (ICLs) [3]. Fig. 1 illustrates a typical 3D system integration using ICLs (as adopted in [4]). Here, planar inductors are fabricated in the upper back-end-ofline (BEOL) interconnect layers in each stacked die, allowing transmission of $\mathrm{AC}$ data or power via electromagnetic (EM) coupling. These systems can therefore make use of standard CMOS processes (without any additional processing steps) resulting in reduced fabrication costs compared to using TSVs.

Both inductive coupling data and power links are often criticised for their inferior power efficiency when compared with TSVs, therefore, when adopting ICLs in 3D-ICs, it is essential that the utilised inductor geometries are optimised. Electrical

${ }^{1}$ Available on-line at http://users.ecs.soton.ac.uk/bjf1g13/coil3d/.

Experimental data used in this paper can be found at DOI:10.5258/SOTON/D0312 (http://doi.org/10.5258/SOTON/D0312).

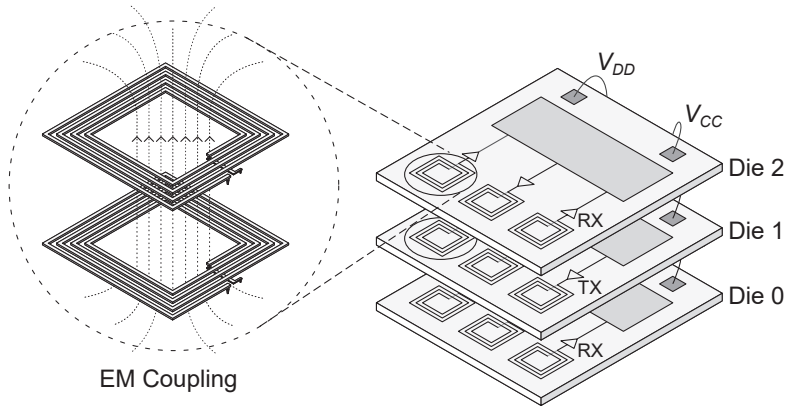

Fig. 1: Illustration of a typical 3D integration using inductive coupling links [4].

simulation of ICLs is not straightforward and requires the system's EM characteristics to be converted into equivalent circuit models that can be handled by typical simulators (e.g. SPICE). This is usually performed using comprehensive finite element analysis (FEA) tools. Solvers using FEA, however, can take several hours to converge, even when analysing a single geometry [5]. In addition to this, the results must be manually converted into lumped electrical models for integration with SPICE. Due to this, finding an inductor pair with optimised geometries (which typically necessitates analysing thousands of subtly different inductor geometries) is extremely computationally expensive, if not impossible. This paper will address this issue, presenting a $C A D$-tool for the Optimisation of Inductive coupling Links for 3D-ICs (COIL3D). COIL-3D uses simplified semi-empirical mathematical models (proposed in this paper) to quickly and accurately determine the performance of a particular inductive coil pair, and hence determine optimised inductor geometries (and associated electrical models), given a set of realistic design constraints. The main contributions of our work include:

- Proposing formulations of the design optimisation problems describing inductive coupling power and data links in 3D-ICs.

- Presenting a set of semi-empirical expressions for quickly evaluating the fundamental electrical parameters of a given ICL.

- Proposing an optimisation flow for determining optimised ICL geometries in 3D-ICs, where both data transmission and power delivery applications are considered.

- Validating the proposed semi-empirical expressions against existing approaches and finite element (FE) solvers (demonstrating that the proposed models achieve 
an average accuracy within 9.1\%). Evaluation of the optimisation flow is also performed (demonstrating a search time reduction of 26 orders-of-magnitude compared to exhaustive linear searches).

\section{BACKGROUND AND RELATED WORKS}

Inductive coupling links exploit the coupled magnetic field between transmit $(\mathrm{Tx})$ receive $(\mathrm{Rx})$ coils and hence facilitate communication of data without physical connection between dies, making them a promising choice for cost-effective (yet, high performance) 3D integration [6]. Whilst interest in the 3D-IC design space is fairly recent, inductive coupling is not a new technology with applications in many areas such as wireless charging, RFID and bio-implanted sensors. Its use in 3D-IC provides a new set of challenges when compared with these historical applications, with a stronger focus on power and area efficiency, commonly with the objective of maximising power efficiency given a specific area constraint.

Evaluation of inductive links is typically performed using comprehensive FEA software packages such as CST Studio or Ansys HFSS. These solvers provide high accuracy, however often take hours to converge at a solution and require manual curve fitting to translate the results to a SPICE model. Alternative solvers include the partial element equivalent circuit (PEEC) method (used in [6] for analysing inductive coupling links in 3D-IC). PEEC solvers, however, still often take a long time to converge at a solution [5], making them too slow for use in optimisation.

More rapid solvers include application specific tools, such as SPIRAL and ASITIC [7], developed for on-chip inductor analysis. These use electrostatic and magnetostatic approximations to provide much faster modelling, but lack the ability to analyse mutual inductance between vertically stacked inductors. Similar, simplified mathematical models are used in [8], where a set of semi-empirical expressions for deriving the power efficiency of an inductive coupling power link are presented. The authors of this paper, however, focus on inductive coupling for bio-medical implants where large inductors are required. As a result, many of the approximations used do not hold true in 3D-IC.

In addition to fast-solvers, for generating optimised link layouts, it is important to establish an efficient optimisation flow. Work [9] by Hsu et al. is the only related research which proposes an optimisation flow for inductive coupling data links. The authors of this paper use an iterative linear search algorithm to determine optimised inductor layouts. To reduce the computational overhead of their approach, however, coils are optimised on an individual basis (considering their selfinductance only) and the coupling between coils is not considered in the flow. This results in solutions often being nonoptimal. The methodology presented in [9] also applies only to ICL data links and would require significant modification for designing ICLs for power delivery. This paper will augment previous work, proposing: (1) a rapid solver based upon semiempirical expressions to quickly and accurately characterise a given link, and (2) a high-speed focussed optimisation flow to

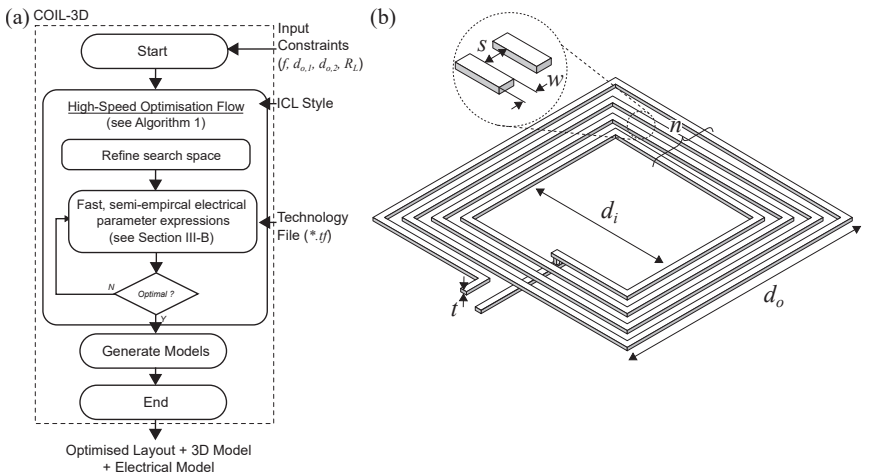

Fig. 2: (a) A high-level operational overview of the COIL-3D tool. (b) Geometric parameters of a square planar coil (outer and inter dimensions $d_{o}$ and $d_{i}$, number of turns $n$, thickness $t_{c u}$, trace width $w$ and spacing $s$ ).

find optimal inductor geometries for use in inductive coupling power transmission or data transmission links. These two contributions are provided together in 'COIL-3D'.

\section{Design Flow Optimisation fOR COIL-3D}

Fig. 2(a) provides a broad operational overview of COIL3D. Beginning with a number of user defined design and technology constraints (outlined in Table I) the tool uses a high-speed optimisation flow (Sect. III-C) and semi-empirical electrical parameters (Sect. III-B) to deliver an optimised inductor layout along with a SPICE model of the link (useful for electrical simulation) and a 3D ICL model (useful for computer-aided IC design or direct import into FEA software for further verification).

As square-spiral inductors offer the highest inductance per unit area (compared to monolithic hexagonal, octagonal and circular inductors [10]), COIL-3D focusses on geometries of this topology. Fig. 2 (b) shows a typical square-spiral inductor and its characteristic geometric parameters: outer and inter dimensions $d_{o}$ and $d_{i}$, number of turns $n$, copper thickness $t_{c u}$ and trace width $w$ and spacing $s$. In an inductive coupling data, or power, link two coils: Coil 1 and Coil 2 (Tx and Rx) are considered, denoted by corresponding subscripts.

For modelling the ICL, COIL-3D uses the lumped equivalent ICL model, proposed in [11] shown in Fig. 3. Here, $L_{i}$ and $R_{i}$ are the inductance and resistance of each coil, $C_{i}$ is the inter-winding capacitance, and $M$ is their mutual inductance.

\section{A. Optimisation Problem Formulation}

ICLs can be used for both data [4] and power transmission [12]. COIL-3D, therefore facilitates two default objective functions, (1) for maximising power delivery efficiency between tiers and (2) for maximising efficiency whilst signalling data.

1) Maximising Power Delivery Efficiency: The power delivery efficiency between tiers, $\eta_{\text {pow }}$, can be directly evaluated considering the equivalent circuit model in Fig. 3. In the case that the link is being used for power transfer, the optimisation problem can be formulated as: 


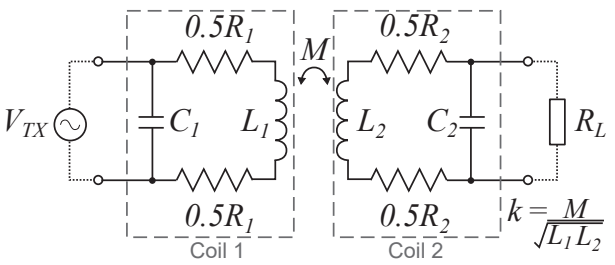

Fig. 3: Equivalent circuit model of an ICL [11] which assumes that each coil can be accurately modelled by its resistance, $R$, capacitance, $C$, and inductance $L$.

$$
\begin{array}{ll}
\text { maximise } & \eta_{\text {pow }}=\frac{\omega^{2} M^{2} R_{L}}{\zeta+R_{1}\left(R_{L}+R_{2}\right)^{2}}\left(\text { See footnote }{ }^{2}\right) \\
\text { subject to } & w_{i}>w_{\text {min }}, s_{i}>s_{\min } \text { (fabrication constraint) } \\
& n_{i}\left(w_{i}+s_{i}\right)<d_{o, i} / 2 \text { (physical constraint) }
\end{array}
$$

where $\quad n_{1}, n_{2} \in \mathbb{Z}+$, and $w_{1}, s_{1}, w_{2}, s_{2} \in \mathbb{R}+$

Here, the constraint $n_{i}\left(w_{i}+s_{i}\right)<d_{o, i} / 2$ ensures that the parameters describe a realisable coil layout.

2) Maximising Data Transfer Efficiency: To derive an objective function for data transmission, the ICL transceiver design must be considered. ICL transceivers, typically use an $\mathrm{H}$-Bridge transmitter circuit to encode data with current pulses which are received and de-coded by a sense-amplifier flip-flop [11]. In ICL systems, the limiting bandwidth factor is the Rx coil capacitance $\left(C_{2}\right)$ which causes inter-symbol interference (ISI) due to ringing in the receiver [11]. Broadly, in order to mitigate this ISI, the time constant $\left(R_{2} C_{2}\right)$ in the receiver coil should be less than $1 / f$, providing an additional optimisation constraint. In this case, rather than considering the power delivery efficiency, $n_{\text {pow }}$ we consider the transfer efficiency $\eta_{d a t}$ equal to $V_{R_{L}} / V_{T x}$ which can be also determined from Fig. 3 . Therefore, for inductive coupling data links, the optimisation problem can be formulated as:

$$
\begin{array}{ll}
\text { maximise } & \eta_{\text {dat }}=V_{R_{L}} / V_{\mathrm{Tx}}\left(\text { See footnote }{ }^{3}\right) \\
\text { subject to } & w_{i}>w_{\min }, s_{i}>s_{\min } \text { (fabrication constraint) } \\
& n_{i}\left(w_{i}+s_{i}\right)<d_{o, i} / 2 \text { (physical constraint) } \\
& R_{2} C_{2}<1 / f \text { (bandwidth constraint) }
\end{array}
$$$$
\text { where } \quad n_{1}, n_{2} \in \mathbb{Z}+\text {, and } w_{1}, s_{1}, w_{2}, s_{2} \in \mathbb{R}+
$$

\section{B. Semi-Empirical Electrical Parameter Expressions}

To realise this design flow, a method of quickly and accurately deriving $L, R, C$ and $M$ for a particular coil must be established. In COIL-3D, a set of rapid semi-empirical expressions outlined in the following subsections are used.

1) Coil Self Inductance $(L)$ and Capacitance $(C)$ : To calculate $L$ the current sheet approximation expression proposed by Mohan et al. is used, as shown in Eqn. 1 [10].

$$
L=\frac{1.27 \mu_{0} n^{2}\left(d_{o}+d_{i}\right)}{4}\left[\ln \left(\frac{2.07}{\phi}\right)+0.18 \phi+0.13 \phi^{2}\right]
$$

TABLE I: COIL-3D parameters and their sources.

\begin{tabular}{ccc}
\hline Parameter & Description & Source \\
\hline$d_{o, i}$ & Maximum Coil $i$ Outer Length & User Input \\
$f$ & Operating Frequency & User Input \\
$R_{L}$ & Receiver Input Impedance & User Input \\
$C_{i, \max }$ & Maximum Coil $i$ Capacitance & User Input \\
$R_{i, \max }$ & Maximum Coil $i$ Resistance & User Input \\
$w_{\min }$ & Minimum Track Width & Technology File $(* . t f)$ \\
$s_{\min }$ & Minimum Track Spacing & Technology File $(* . t f)$ \\
$D$ & Communication Distance & Technology File $(* . t f)$ \\
$g_{x, y}$ & Grid Resolution (x and y) & Technology File $(* . t f)$ \\
$L_{i}$ & Coil $i$ Self-Inductance & Calculated by COIL-3D \\
$R_{i}$ & Coil $i$ Resistance (between terminals) & Calculated by COIL-3D \\
$C_{i}$ & Coil $i$ Capacitance (between terminals) & Calculated by COIL-3D \\
$M$ & Mutual Inductance (between coils) & Calculated by COIL-3D \\
\hline
\end{tabular}

where $\phi$ is the coil's fill-factor given by $\left(d_{o}-d_{i}\right) /\left(d_{o}+d_{i}\right)$. For calculating $C$, Eqn. 2 is used, where $\kappa$ is an empirical constant.

$$
C=\kappa \varepsilon_{0} \varepsilon_{\mathrm{SiO}_{2}} w t_{c u}\left\{\sum_{z=0}^{2 n-1} 2\left[d_{o}-z(w+s)\right]\right\}^{-1}
$$

2) Coil Resistance $(R)$ : Other works propose a variety of methods for estimating the resistance of planar spiral inductors, however the most commonly used model is the resistivity equation incorporating high-frequency conduction loss. Whilst this provides a reasonable approximation for larger coils, when considering micron scale coils (used in 3D-IC) the yielded values are typically too low, due to the proximity effect increasing the apparent resistance by a factor $k_{p}$ (known as the proximity factor). In depth work, deriving differential equations for calculating $k_{p}$ is available [13], however these expressions are not strictly solvable, making evaluation in software very computationally expensive. As such, in COIL$3 \mathrm{D}$, values of $k_{p}$ are empirically pre-determined (as a function of $w$ and $s$ ) and stored in a lookup table for use at runtime. Using these values, coil resistance is determined by Eqn. 3 .

$$
R=k_{p}(w, s) \sum_{z=0}^{2 n-1} 2\left[d_{o}-z(w+s)\right] \frac{1}{2 w t_{c u}} \cdot \sqrt{\frac{\pi f \mu_{0}}{\sigma_{c u}}}
$$

3) Mutual Inductance ( $M)$ between Tx and Rx Coils: For calculating $M$, an expression can be derived from Maxwell's equation for the mutual inductance between two air-cored loops. If an assumption is made that the two communicating coils are perfectly vertically aligned, the mutual inductance between two loops over a distance $D$ is given by:

$$
M_{a, b, D}=\frac{2 \mu_{0}}{\alpha} \sqrt{a b}\left[\left(1-\frac{\alpha^{2}}{2}\right) K(\alpha)-E(\alpha)\right]
$$

where $a$ and $b$ are the radii of the two loops and $\alpha=$ $2 \sqrt{a b /\left[(a+b)^{2}+D^{2}\right]}$. Here $K(\alpha)$ and $E(\alpha)$ are the complete elliptic integrals of the first and second kind respectively. As the structure of a planar spiral inductor is not a single loop, moreover a set of $n$ concentric interconnected turns, the

${ }^{2} \zeta=R_{L}{ }^{2} \cdot\left[\left(\omega^{4} C_{2}{ }^{2}\left(R_{2} M^{2}+R_{1} L_{2}^{2}\right)\right)+\omega^{2}\left(R_{1}\left(C_{2}^{2} R_{2}^{2}-2 C_{2} L_{2}\right)+\left(L_{2}^{2} R_{1}+M^{2}\left(R_{L}+R_{2}\right)\right)\right)\right]$

${ }^{3} V_{R_{L}} / V_{\mathrm{Tx}}=1 /\left(1+j \omega R_{2} C_{2}\right) \times j \omega k \sqrt{L_{1} L_{2}} \times 1 /\left(R_{L}\left(1-\omega^{2} L_{1} C_{1}\right)+R_{1}+j \omega\left(C_{1} R_{1} R_{L}+L_{1}\right)\right)$ 


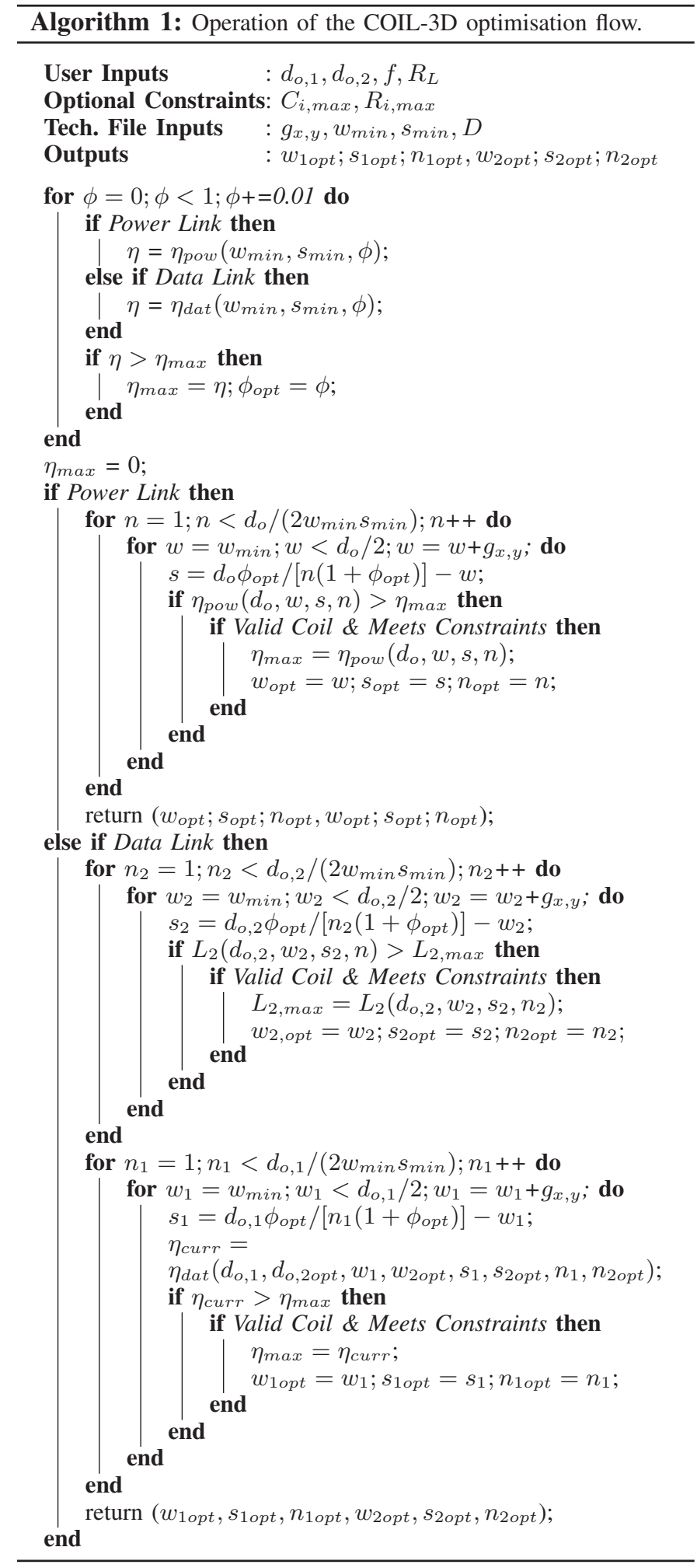

approximation is often made that the total mutual inductance is the cumulative summation of mutual inductance between each turn of the Tx coil and every turn of the Rx coil [14], leading to Eqn. 5.

$$
M_{\text {tot }}=g \sum_{i=1}^{n_{1}} \sum_{j=1}^{n_{2}} M\left(a_{i}, b_{j}, D\right)
$$

Previous works [8], [15], [16] suggest the introduction of a correction factor here, $g$ which takes the value $g \approx 1.1$. Although practical validation found this model to be reasonably accurate for coils with fewer than 10 turns, when considering inductors with $n>10$, the model accuracy deteriorates. This is because as $n$ increases, the assumption of equal coupling between every turn of each coil, introduces larger error. In COIL-3D, this degradation in coupling is incorporated by a scaling factor, $r_{i, j}$ corresponding to the Pythagorean distance between turns, normalised with respect to a pair in perfect vertical alignment, such that:

$$
r_{i, j}=\frac{1}{D} \sqrt{[(i-j) \cdot(w+s)]^{2}+D^{2}}
$$

We therefore replace the single correction factor $g$ as shown in Eqn. 7 where $k_{f}$ is an empirical constant.

$$
M_{\text {tot }}=\sum_{i=1}^{n_{1}} \sum_{j=1}^{n_{2}}\left(\frac{1}{r_{i, i}}\right)^{k_{f}} M\left(a_{i}, b_{j}, D\right)
$$

\section{Complexity Analysis}

Having presented expressions for deriving $\eta_{\text {pow }}$ and $\eta_{d a t}$, an optimisation flow for determining best performing ICL layouts must be established. Applying exhaustive linear optimisation to the problems outlined in (1) and (2) results in an extremely high time complexity $\left(O\left(n^{6}\right)\right)$. To reduce the computational overhead of the search problem, COIL-3D introduces an additional parameter, the fill factor $(\phi)$. Optimised inductor layouts typically have a fill factor around 0.4 [8] (however in COIL$3 \mathrm{D}$, a bespoke optimised value is determined at the beginning of each run) and therefore centring the search around a fixed fill-factor avoids the extra computational overhead incurred whilst evaluating probabilistically non-optimal designs e.g. where $\phi=0.9$. By adding this constraint the solution space can be refined, and the time complexity reduced to $O\left(n^{4}\right)$.

To further speed-up the algorithm whilst searching for inductive coupling data links, optimisation is divided into two discrete stages: Rx coil optimisation, and Tx coil optimisation. From the ICL transfer equation (footnote 3), it can be observed that $\eta_{\text {dat }}$ will be maximised when $L_{2}$ is maximised, provided that the time constant $R_{2} C_{2}$ (discussed earlier) in the denominator of the first term is constrained. Therefore, the $\mathrm{Rx}$ coil is initially optimised to provide maximum $L_{2}$ within the imposed bandwidth constraints. The Tx coil is then optimised for $\eta_{d a t}$ which considers the mutual inductance between the pair. Dividing the flow in this way reduces the time complexity of the search to $O\left(n^{2}\right)$, without compromising on accuracy. To further reduce the time complexity of the algorithm whilst searching for inductive coupling power links, it can be noted that $\eta_{\text {pow }}$ will be maximised when $d_{o, 1}=d_{o, 2} w_{1}=w_{2}$, $s_{1}=s_{2}$ and $n_{1}=n_{2}$. Therefore, the number of optimisation parameters, and hence time complexity, is halved to $O\left(n^{2}\right)$, again without compromising on accuracy.

Combining these improvements, Algorithm 1 shows the operation of the COIL-3D optimiser. First, an optimal value of $\phi$ is found using the efficiency equations from Sect. III-A, 


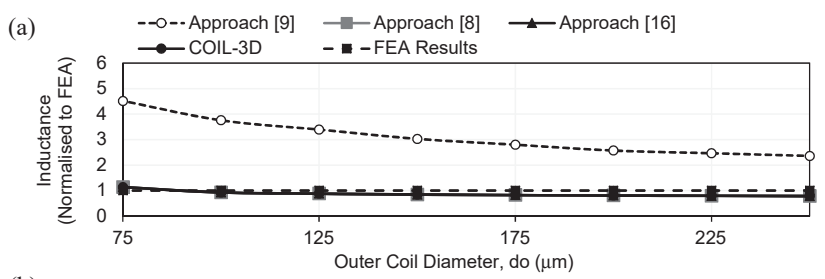

(b)

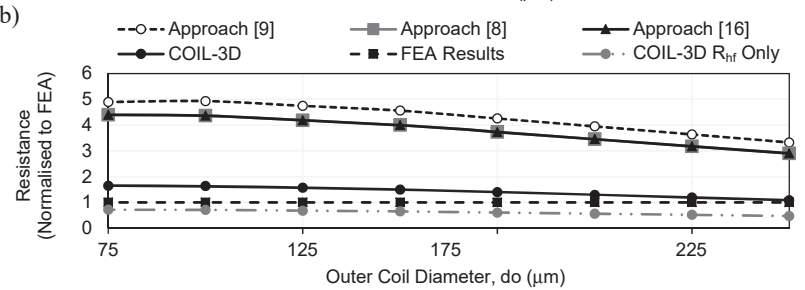

Fig. 4: (a) Inductance and (b) Resistance extraction accuracy as $d_{o}$ varies $(n=8, w=s=1 \mu \mathrm{m}, f=1 \mathrm{GHz})$

with minimum track width and spacing to provide maximum resolution. $\phi_{\text {opt }}$ is then used to refine the search space and, incorporating the simplifications outlined above, the COIL-3D optimiser exhaustively searches all parameters to guarantee a globally optimal solution.

\section{EXPERIMENTAL RESUlts}

\section{A. Experimental Set-up}

The proposed methodology (COIL-3D) was implemented in $\mathrm{C}++$ and experimentally validated on a quad core Intel CPU. COIL-3D was compared with existing optimisation flows, benchmarking the accuracy of each approach against finite element analysis results obtained using CST MW Studio [17] (configured to use a frequency-domain solver, with a mesh resolution of 10 cells-per- $\lambda$ ). Evaluation of both electrical parameter expression accuracy (Sect. IV-B1 \& 2) and algorithmic search accuracy (Sect. IV-B3) was performed for a variety of use-case scenarios across a range of operating frequencies.

\section{B. Results and Evaluations}

1) $R, L$ and $M$ Expression Accuracy: Initially the accuracy of the proposed semi-empirical expressions for $R, L$ and $M$ were evaluated for a range of coil sizes at $1 \mathrm{GHz}$. Figures 4 (a) and (b) show the inductance and resistance evaluation accuracy (respectively) of COIL-3D in addition to approaches [8], [9] and [16]. It can be observed that the COIL-3D solver consistently evaluates $R$ and $L$ accurately (< $17 \%$ error) across a range of coil diameters. The resistance extraction accuracy of COIL-3D much exceeds that of existing approaches by incorporating both skin and proximity effects. For calculating inductance, the current sheet approximation approach used in COIL-3D (and [8], [16]) can be observed to significantly outperform the data-fitted monomial expression used in [9] in the examined cases. Fig. 5 shows similar results, for mutual inductance extraction accuracy as $n$ varies (again, the accuracies of approaches [8] and [16] have been included for comparison, however [9] has been omitted as detailed derivation of mutual inductance is not presented in this work). It can be observed that the proposed mutual inductance model

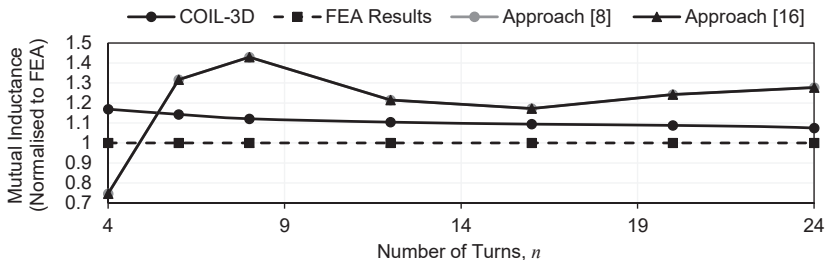

Fig. 5: Mutual inductance extraction accuracy as $n$ varies ( $d_{o}=300 \mu \mathrm{m}, w=1.5 \mu \mathrm{m}, s=1 \mu \mathrm{m}$ for both coils).

improves upon existing approaches, particularly in cases where $n>10$, as previously hypothesised.

2) $\eta_{\text {pow }}$ Calculation Accuracy: As further verification of the expressions used in COIL-3D, the power transmission efficiencies (combining parameters $R, L, M$ and $C$ ) of 50 randomly generated ICL geometries were evaluated, for frequencies between 0.25 and $1.25 \mathrm{GHz}$. To ensure validity of the experiment, the randomly generated parameters were constrained to mimic those typical of ICL applications $(150 \mu \mathrm{m}<$ $d_{o}<400 \mu \mathrm{m}, 2<n<20$, and $\left.0.2 \mu \mathrm{m}<w \& s<10 \mu \mathrm{m}\right)$. Fig. 6 illustrates the calculation accuracy (benchmarked against FEA) for each link geometry using both COIL-3D, and approach [8] (the only other work detailing expressions for $R, C, L$, and $M$ in full). The calculation error was found to be less than $29.2 \%$ for the entire sample, with a considerably lower average error of $9.1 \%$. The results also show that the improved semi-empirical expressions presented in this paper perform better than those in [8] by $59.1 \%$ on average.

3) Algorithm Evaluation: The effectiveness of the COIL3D optimisation algorithm was also explored. In COIL-3D, the search complexity is reduced by constraining coil fill-factor to a single value and therefore, the accuracy of the proposed search approach is dependent on the ability to pre-determine optimal fill-factors correctly. Due to the impossibly high compute resource associated with analysing every possible inductor geometry using FEA as a comparison benchmark, the effectiveness of each algorithm is hard to quantify, however, a selection of contrived tests on a large grid $\left(g_{x, y}=w_{\min }=\right.$ $s_{\text {min }}=0.8 \mu \mathrm{m}$ and $d_{o}=80 \mu \mathrm{m}$ ) were performed. A total of 20 optimisation challenges were run, each with different die separations and operating frequencies to establish the search accuracy of each approach (COIL-3D, [8] and [9]). Fig. 7 shows the results of these tests. Here, it can be observed that COIL-3D finds optimal (or near-optimal) solutions much more consistently than the other two approaches. This is likely due to the consideration of mutual inductance in the optimisation flow (which is not incorporated in [8] or [9]).

4) Overhead Evaluation: Finally, the execution overheads were evaluated. Table II shows the average time taken to evaluate the efficiency of a single ICL using COIL-3D, approach [8] and FEA. Whilst COIL-3D is not the fastest of the three explored in this paper, it is approximately 6 orders-of-magnitude faster than FEA, whilst maintaining a reasonable average accuracy $(<10 \%)$. Table III shows the estimated/simulated total optimisation times for the various solver/optimiser combinations, assuming a $0.1 \mu \mathrm{m}$ grid and an 


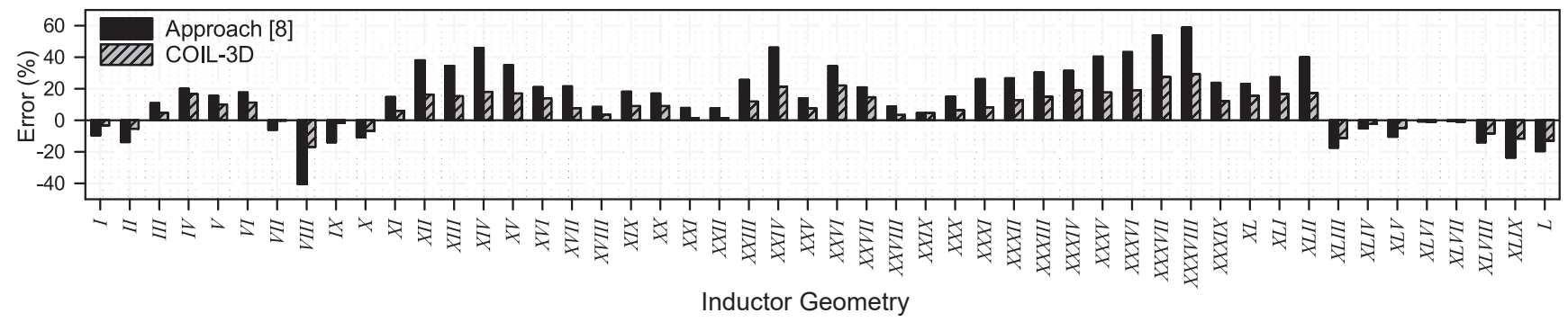

Fig. 6: Accuracy comparison between COIL-3D and approach [8] for 50 randomly generated ICL geometries.

TABLE II: Execution overheads of the proposed electrical parameter expressions (when evaluating $\eta_{\text {pow }}$ ) compared with existing approaches.

\begin{tabular}{ccc}
\hline Solver & $\begin{array}{c}\text { Average Execution } \\
\text { Time (per geometry) }\end{array}$ & $\begin{array}{c}\text { Normalised Average } \\
\text { Error (\%) }\end{array}$ \\
\hline FEA Solver [17] & $5,450 \mathrm{~s}$ & $0 \%$ \\
\hline Simplified Expressions in [8] & $0.008 \mathrm{~s} \mathrm{~s}$ & $22.3 \%$ \\
\hline COIL-3D Solver & $\mathbf{0 . 0 1 5} \mathrm{s}$ & $\mathbf{9 . 1 \%}$ \\
\hline
\end{tabular}

TABLE III: Total optimisation time using COIL-3D and other approaches.

\begin{tabular}{ccc}
\hline Approach & ICL Type & $\begin{array}{c}\text { Predicted }^{\dagger} \text { /Actual } \\
\text { Execution Time }^{-12}\end{array}$ \\
\hline FEA [17] + exhaustive linear search & Power or Data & $10^{22}$ Years $^{\dagger}$ \\
\hline $\begin{array}{c}\text { FEA Solver [17] with proposed refined } \\
\text { search algorithm (proposed for COIL-3D) }\end{array}$ & Power or Data & 518 Years $^{\dagger}$ \\
\hline $\begin{array}{c}\text { Semi-empirical expressions (proposed for } \\
\text { COIL-3D) with exhaustive linear search }\end{array}$ & Power or Data & $10^{18}$ Years $^{\dagger}$ \\
\hline Iterative Optimisation Flow [8] & Power Only & 124 Mins \\
\hline $\begin{array}{c}\text { ThruChip Inductive Coupling Channel } \\
\text { Design Optimisation Flow [9] }\end{array}$ & Data Only & 12.9 Mins \\
\hline $\begin{array}{c}\text { COIL-3D (Semi-empirical solver with } \\
\text { refined search algorithm) }\end{array}$ & Power or Data & $\mathbf{2 4 . 9 ~ M i n s . ~}$ \\
\hline
\end{tabular}

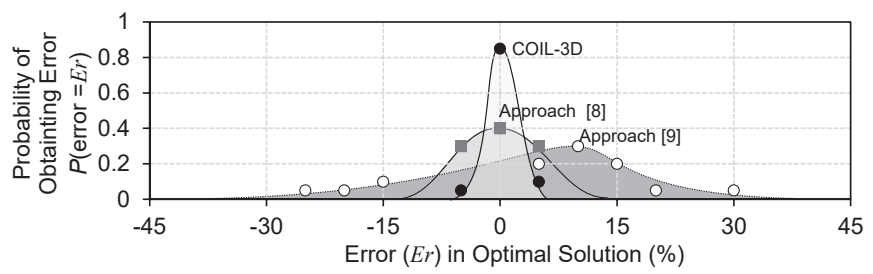

Fig. 7: Optimisation search effectiveness (when maximising $\eta_{d a t}$ ) for COIL-3D and existing approaches.

area constraint of $300 \mu \mathrm{m}$. Here, it can be observed that the COIL-3D tool arrives at optimised geometries faster than each of the analysed alternatives, with the exception of approach [9] which considers the two inductors independently and hence suffers from low accuracy (as discussed in Sect. IV-B3).

\section{CONClusion}

This paper has presented COIL-3D, a computational design tool for generating optimised inductive coupling links in 3D-IC. Motivated by the significant computational overhead incurred whilst using FEA, this work has presented a selection of semi-empirical mathematical equations to quickly and accurately compute the efficiency of a given link, and a corresponding design flow to find optimised ICL geometries for either power or data transmission. Experimental results show that the proposed approach is up to 26 orders-ofmagnitude faster than finite element methods (in conjunction with exhaustive linear optimisation), whilst still maintaining high computational accuracy (within 9.1\%). Additionally, the models in COIL-3D were found to outperform existing semiempirical methods by approximately $59.1 \%$. COIL-3D will be made publicly available in the near future, as a software tool for integration with inductive coupling 3D-IC CAD flows.

\section{REFERENCES}

[1] P. Lindner et al., "Key enabling processes for more-than-Moore technologies," in IEEE Int. SOI Conf. (SOI), 2012.

[2] B. Swinnen et al., "3D integration by $\mathrm{Cu}-\mathrm{Cu}$ thermo-compression bonding of extremely thinned bulk-Si die containing 10um pitch through-Si vias," in Internat. Electron Devices Meeting, 2006.

[3] T. Kuroda, "Wireless proximity communications for 3D system integration," in IEEE Int. Workshop on RF Integration Technology, 2007.

[4] D. Ditzel and T. Kuroda, "Low-cost 3D chip stacking with ThruChip wireless connections," in IEEE Hot Chips 26 Symp.(HCS), Aug 2014.

[5] J. Park et al., "Efficient calculation of inductive and capacitive coupling due to electrostatic discharge using PEEC method," IEEE Trans. on EM Compat., vol. 57(4), pp. 743-753, 2015.

[6] T. Vali et al., "Full-wave modeling of inductive coupling links for lowpower 3D system integration," in IEEE Int. Symp. EM Compat., 2013.

[7] A. M. Niknejad and R. G. Meyer, "Analysis and optimization of monolithic inductors and transformers for RF ICs," in Custom Integrated Circuits Conf. (CICC), 1997.

[8] U. M. Jow et al., "Design and optimization of printed spiral coils for efficient transcutaneous inductive power transmission," IEEE Trans. on Biomedical Circuits and Systems, vol. 1(3), pp. 193-202, 2007.

[9] L. C. Hsu et al., "Analytical thruchip inductive coupling channel design optimization," in 21st Asia and South Pacific Design Automation Conf. (ASP-DAC), 2016, pp. 731-736.

[10] S. Mohan et al., "Simple accurate expressions for planar spiral inductances," IEEE J. Solid-State Circuits, vol. 34(10), pp. 1419-24, 1999.

[11] N. Miura et al., "Analysis and design of inductive coupling and transceiver circuit for inductive inter-chip wireless superconnect," IEEE Jour. of Solid-State Circuits, vol. 40(4), pp. 829-37, 2005.

[12] S. Han and D. D. Wentzloff, "Wireless power transfer using resonant inductive coupling for 3d integrated ics," in IEEE Int. 3D Systems Integration Conf. (3DIC), Nov 2010, pp. 1-5.

[13] G. S. Smith, "Proximity effect in systems of parallel conductors," Journal of Applied Physics, vol. 43(5), pp. 2196-2203, 1972.

[14] M. F. Chang et al., "RF interconnect for inter and intra-chip communications," Proc. of the IEEE, vol. 89(4), no. 4, pp. 456-66, 2001.

[15] M. Farran et al., "Design, simulation and testing of planar spiral coils for the time gated interrogration of quartz resonator sensors," in Proc. 28th European Conf. on Modelling and Simulation (ECMS), 2014.

[16] B. Noroozi and B. I. Morshed, "PSC optimization of $13.56-\mathrm{MHz}$ resistive wireless analog passive sensors," IEEE Trans. on Microwave Theory and Techniques, no. 99, pp. 1-8, 2017.

[17] C. S. Technology, "CST microwave studio," CST MICROWAVE STUDIO, 2015. [Online]. Available: https://www.cst.com/products/cstmws 